\title{
Applied research status and its key technologies development of the electromagnetic linear actuator
}

\author{
Ningxia Yin ${ }^{1}$, Hongchen Pang ${ }^{1}$, Guanghui $\mathrm{Li}^{1,}$ a and Shenjie $\mathrm{Li}^{2}$ \\ ${ }^{1}$ Engineering College, Guangdong Ocean University, 524088 Zhanjiang, China \\ ${ }^{2}$ Material Management Centre, China Oilfield Services Limited (Zhanjiang Branch), 524057 Zhanjiang, China
}

\begin{abstract}
Firstly, this paper briefly describes the origin of the electromagnetic linear actuator which research status and development are summarised. Secondly, it describes the principle, the characteristics of the moving-coil linear motor and the Moving-magnet linear motor. Thirdly, it analyses and summarizes the key technologies of the electromagnetic linear actuator. One key problem is the matching between electromagnetic force and the load. The other is the influence mechanism of the thermal load on dynamic characteristics. This paper can provide some reference.
\end{abstract}

Keywords: electromagnetic linear actuator; matching; thermal load; development trend.

\section{Introduction}

With the decreasing of energy sources and aggravating of worldwide environment, energy-saving and emission reduction have become two themes of social development in the 21st century [1]. As a new executive body, the electromagnetic linear actuator which combines drive and transmission has many advantages, such as short transmission chain, mechanical simplicity, low friction, small vibration, low noise and high efficiency [2-5]. It meets the technical requirement of mechanical drive , transmission parts and unit in China's national policy for medium and long-term scientific development (20062020)[7]. With the ever-growing demands of energy saving and emission reduction, electromagnetic linear actuator is attracting more and more attention [6-8]. Now it has been used in many technical fields and industries such as high-grade numerically-controlled machine, special processing machine tool, robot, free piston engine, linear compressor and so on [8, 11-15].

\section{Origin and development of the electromagnetic linear actuator}

\subsection{Origin of the electromagnetic linear actuator}

The prototype of the electromagnetic linear actuator were developed by Wheatstone in 1840[16, 17], which were almost the same time with the electric rotating machinery. Fig. 1 shows the process of rotary into linear mot [18]. Although it didn't succeed at that time, it brought a new conception for successors in the future.

\footnotetext{
${ }^{\mathrm{a}}$ Corresponding author : ligh2009@126.com
} 


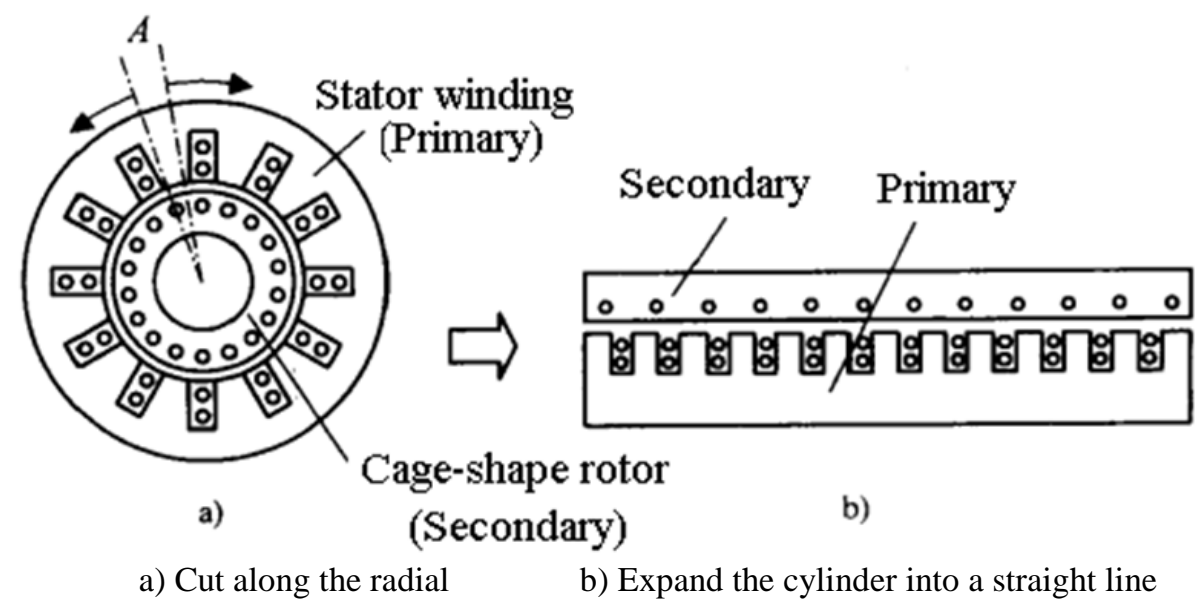

Figure 1. Process of rotary into linear motor

\subsection{Development of the electromagnetic linear actuator}

Since 1840, the electromagnetic linear actuator has experienced three periods which are experiment exploration, application development and commercialization respectively [16]. Especially since the 1990's, the electromagnetic linear actuator has been used more and more widely.

The electromagnetic linear actuator can be put into two categories: moving-coil linear motor (the coil moves) and moving-magnet linear motor (the permanent magnet moves). Fig.2 shows the two linear motors.

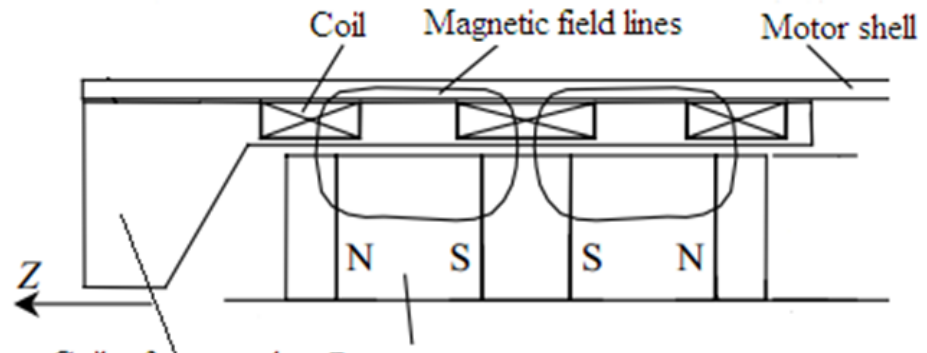

Coiler framework Permanent magnet

a) Moving-coil linear motor [19]
Stator core Permanent magnet

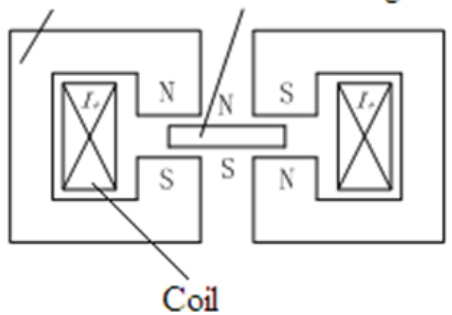

b) Moving-magnet linear motor[20]

Figure 2. Two linear motors

The major differences of the moving-coil linear motor and the moving-magnet linear motor are shown in Table 1.

Table 1. Differences of the moving-coil and the moving -magnet.

\begin{tabular}{ccc}
\hline Difference & Moving-coil & Moving- magnet \\
Motion parts & Coil & Magnet \\
Complexity & Relatively simple & Complex \\
Motion mass & Low & Large \\
Frequency & High & Low \\
Cost & Low & High \\
Power density & Low & High \\
\hline
\end{tabular}




\section{Key problems of the electromagnetic linear actuator}

The electromagnetic linear actuator is mainly used for short-stroke and high-frequency linear reciprocating motion, as a result of that kinetic characteristic of short-range and high-frequency can be obtained through control the current of the coil [17]. As the executing structure, the movement of the electromagnetic linear actuator is in the non-steady state of acceleration, deceleration and reversal. So there are two key problems to be solved which are the matching between electromagnetic force and the load, the influence mechanism of the thermal load on dynamic characteristics.

\subsection{Coupling law between electromagnetic force and load characteristics}

Due to short motion chain, there is no conversion unit, transferring and buffering effect. Load unit directly effects on the moving parts. It is very important that the coupling law between electromagnetic force and load characteristics on the energy conversion, transmission and motion control. So seeking the coupling law between electromagnetic force and load characteristics has been one of the key problems to be solved

The coupling of the electromagnetic field, the electric field and field force of the mechanical movement is the main problem. In addition to the equations which describe the electrical circuit control and the movement of the mechanism can be embedded in the finite element methods, there is another problem to be solved.

Well-controlled electromagnetic force is one of the basic conditions for the coupling of electromagnetic linear actuator with external load. In the past several years, worldwide scholars have studied the solenoid structure of the electromagnetic linear actuator which can achieve precise control widely and deeply. Among all the solenoid structures, permanent magnet synchronous linear motor is proposed early [21]. Due to its inherent slot effect and end effect, there is thrust fluctuation which will affect its controlled characteristics. One significant advantage of the moving-coil electromagnetic linear motor is that the electromagnetic force is better linear with the electric current, so it is convenient for decoupling precise control [22]. As a result it is mainly used for small and mediumsized thrust occasions. But for the high thrust occasion, the moving-magnet linear motor is proposed [23]. However, the system natural frequency will be affected by the inherent characteristics of the magnetic spring, which make the electromagnetic system and the mechanical system have strong coupling characteristics [24].

\subsection{Influence mechanism of the thermal load on dynamic characteristics}

The thermal load is the heat loss incurred during the electromagnetic linear actuator process. There is no defined standard to definitite the loss form of the electromagnetic linear actuator which is shown as Fig. 3 [25]. Although the kinetic equations can be established through mechanic analysis and the governing equation of electromagnetic force can be inversely solved, the reverse of the electromagnetic linear actuator is very difficult to control. Because of short-stroke and highfrequency, the control of electromagnetic force can't meet the high requirement of real-time and high accuracy, as a result of which the system kinematics will be seriously damaged, especially the reverse will be advanced or delayed on account of large inertia force. In order to have a stable travel control and frequency control system with lower energy consume, some research is around the coupling of the electromagnetic force and the system dynamics parameters from the perspective of mechanical vibration. Based on the mathematical model of moving magnet linear compressor, the system natural frequency is gotten by Xie through equivalent the compressed gas to a certain damping and stiffness [26]. Kim et al. studied the relatio between the inherent frequency and the coefficiency of performance [27]. 


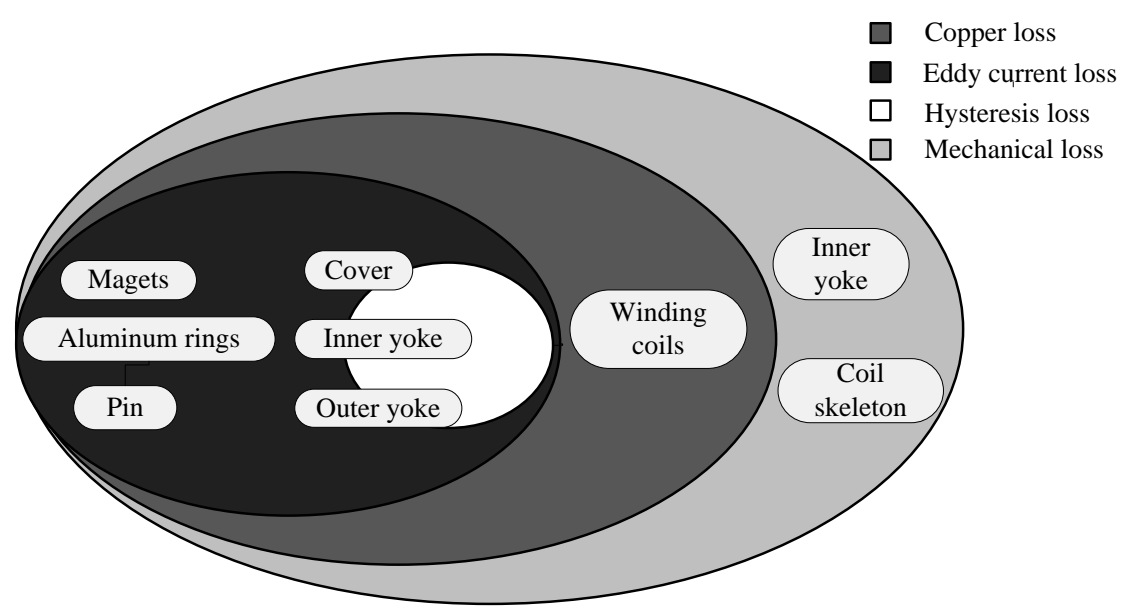

Figure 3. Loss form and distribution of electromagnetic linear actuator [25]

Thus it can be seen that the system works in a state of resonance by making the load equivalent to certain kinetic parameter from the perspective of single free degree vibration. The inherent frequency can be gotten and the electromagnetic force frquency can be ajusted which are consistent with the inherent frequency. But the adaptability of the electromagmetic linear actuator is poor, and the resonance can only work under constant load. Once the load change is uncertain, its equaverlent parameters are varialbe and unpredictable [26].

The heat loss in the course of electromagnetic linear actuator working includes the copper loss caused by the electric current loading method and the core loss caused by magnetic field change. The unsteady motion state results that the electric current loading method and the magnetic field are all high-frequency alternately which will generate a large amount of core loss and temperature rise sharply due to large amount of thermal load affect the electromagnetic properties. Due to high residual magnetic flux density, high magnetic energy product and linear demagnetization curve, the $\mathrm{NsFeB}$ material is widely used in modern permanent magnet motor. However, the material is of high temperature coefficient, poor magnetic thermal stability even demagnetized after a certain temperature, so the magnetic properties decreases dramatically [29, 30]. At the same time, excess temperature is the main cause of deterioration of the electromagnetic linear actuator performance, which will further affect the dynamic characteristic of the electromagnetic linear actuator.

\section{Development trend of the electromagnetic linear actuator}

Much progress in electromagnetic linear actuator is expected in coming years. And the development trend of electromagnetic linear actuator can be summarized in three aspects. One development trend is the application more and more widely. It has been used in many areas such as aerospace, processing plants and internal combustion engine [31]. The proposed electromagnetic linear actuator is expected to be one of the solutions for safe or high performance [32]. The second trend is high-speed and highprecision. High speed and high precision are always goals of motion control [33]. Precision positioning and control are hey technologies at high-speed. The third trend is high-thrust and highcapacity.

\section{Conclusions}

1) With the advantages of short transmission chain, mechanical simplicity, low friction, small vibration, low noise and high efficiency, the electromagnetic linear actuator has been one development trend of the executive body. 
2) On the basis of analysis the features of the electromagnetic linear actuator, the paper sums up that there are two key problems to be solved. One key problem is the matching between electromagnetic force and the load. The other is the influence mechanism of the thermal load on dynamic characteristics.

\section{Acknowledgments}

This study is supported by the Project of Enhancing School with Innovation of Guangdong Ocean University (GDOU2015050225). We would like to thank the sponsors.

\section{References}

1. S. Hu. Design and implementation of the new aeroderivatives power controller[D]. Chengdu: University of Electronic Science and Technology of China, (2010).

2. Y. Fujimoto, T. Kominami, H. Hanmada. Development and analysis of a high thrust force directdrive linear actuator[J]. IEEE Transactions on Industrial Electronics, 56(5):1383-1392, (2009)

3. X. Shi, S. Chang. Extended state observer-based time-optimal control for fast and precise pointto-point motions driven by a novel electromagnetic linear actuator. Mechatronics, 23: 445-451, (2013)

4. M. Jin Chung. Development of High-Speed Response Electromagnetic Linear Actuator Using for Pneumatic Control Valve. Applied Mechanics and Materials, 532: 41-45, (2014)

5. J. Wang, X. Shangguan, Z. Liao. Status and development of linear motor drive divided compressor. Compressor Technology, 1998(1): 46-48.

6. L. Chen, L. Li. Development of the linear motor and its key technologies for compressors. Proceedings of the CSEE, 33(15):52-68 (2013).

7. State Council of the People's Republic of China. China's national policy for medium and longterm scientific development (2006-2020). (2006).

8. J. Dai, S. Chang. L. Liu. Optimization analysis of electromagnetic linear actuator's radial array permanent magnets. International Journal of Applied Electromagnetics and Mechanics., 47: 441451, (2015).

9. C. Liu, Y. Chiu. C. Hwang. Designs and Feasibility Assessments of an Integrated Linear Electromagnetic Actuator for Cold Roll Mill Applications. Industry Applications, IEEE Transactions on. 1, (2016)

10. P. Zhao, Y. Chong, A. Zhao, et al. A rapid infusion pump driven by micro electromagnetic linear actuation for pre-hospital intravenous fluid administration. Proceedings of the Institution of Mechanical Engineers. Part H. 229(2):101-109, (2015)

11. Q. Zhang. Application of linear motor direct drives system in high speed and precision processing. Mechanical \& Electrical Engineering Technology, 35(6):45-47, (2006).

12. Martins, J. Esteves, G.D. Marques and F.P. da Silva, Permanent-magnets linear actuators applicability in automobile active suspensions, IEEE Transactions on Vehicular Technology 55 , 86-94, (2006).

13. H. Lu, J. Zhu, Z. Lin and Y. Guo, An inchworm mobile robot using electromagnetic linear actuator, Mechatronics, 19: 1116-1125, (2009).

14. J. Zhang, Y. Chang, Z. Xing. Fuzzy control of piston displacement of linear refrigerator compressor. Journal of Xi'an Jiaotong University, 43(1): 38-42, (2009).

15. J. Kim, J. Ieong. Performance characteristics of a capacity-modulated linear compressor for home refrigerators. International Journal of Refrigeration, 36(3):776-785, (2013).

16. Y. Ye. Development and trend of domestic \& overseas linear motor technologies. Electric age, 4: 26-29, (2012).

17. Boldea. Linear electric actuators and generators. Cambridge University Press, (1997),

18. Y. Ye. Principle and application of linear motor. Beijing: China Machine Press, (2000),. 
19. X. Xu, L. Quan, X. Wang. Moving coil motor used in servo proportional valve. Proceedings of the CSEE, 30(9):92-96, (2010)

20. M. Lei, W. Dai, Y. Xia. Modelling and analysis of moving-magnet linear motor with no innerteeth for linear compressor. Electric machines and control, 18(11):45-50, (2014).

21. J. Hong, L. Li. Current error vector based prediction control of currents in segment winding permanent magnet linear synchronous motor. Proceedings of the CSEE, 31(30):77-84, (2011)

22. Vadan, H. Balan, R. Munteanu. Study and design of a linear compressor of voice-coil type [J]. Advances in Electrical \& Computer Engineering, 9(2):109-114, (2009).

23. J. Wang, D. Howe, Z. Lin. Design optimization of short-stroke single-phase tubular permanent magent motor for refrigeration applications [J]. IEEE Transactions on Industrial Electronics, 57(1):327-334, (2010).

24. M. Xia, X. Chen. Analysis of resonant frequency of moving magnet linear compressor of Stirling cryocooler. International Journal of Refrigeration, 33(4):739-744, (2010).

25. J. Dai, S. Chang. Loss analysis of electromagnetic linear actuator. International Journal of Applied Electromagnetic and Mechanics, 46(3): 471-482, (2014).

26. J. Xie, X. Li, Z.Xiao. Numerical simulation and analysis of moving-magnet linear refrigerator compressor. Journal of central south university of forestry and technology, 30(8):133-137, (2010).

27. H. Kim, C. Roh, J. Kim, et al. An experimental and numerical study on dynamic characteristic of linear compressor in refrigeration system [J].International Journal of Refrigeration Revue Internationale Du Froid, 32(7):1536-1543, (2009).

28. H. Zhang, L. Zhang, G. Peng, et al. Performance simulation and experiment research on movingmagnet linear oscillation motor. Electric machines and control, 16(4) : 25-29, (2014).

29. R. Tang. Modern peperaturmanent magnet moourtor- theory and desig. Beijing, China Machine Press,(2008).

30. S. Jiang. High-speed permanet magnetsynchronous motor loss analysis and temperature field calculation. Harbin, Harbin Institute of Technology, (2010),

31. N. Sidell, G. W. Jewell. The design and construction of a high temperature linear electromagnetic actuator. Journal of applied physics, (1999), 85(8) :4901-4903.

32. Y. Fujimoto, T. Kominami, H. Hamada. Development and Analysis of a High Thrust Force Direct-Drive Linear Actuator. IEEE transaction on industrial electronics, 56(5) : 1383-1392, (2009).

33. T. Hama;K Sato. High-speed and high-precision tracking control of ultrahigh-acceleration moving-permanent-magnet linear synchronous motor. Precision engineering, 40 : 151-159, (2015). 